\title{
Surgical Management and Functional Outcome of Primary Convexity Subdural Empyema With Inter-hemispheric Extension: A Case Report and Literature Review
}

Moayad Moawia Zain Elabdin Ahmed ( $\sim$ moayadmz@gmail.com)

Aliaa Specialist hospital https://orcid.org/0000-0001-9947-3593

Shahd A.Y Alias

Mukashfi E. A. Ali

Zakaria Ibrahim Mohammed

\section{Case report}

Keywords: Subdural empyema, Magnetic resonance imaging, inter-hemispheric subdural empyema

Posted Date: June 8th, 2020

DOI: https://doi.org/10.21203/rs.3.rs-33327/v1

License: @) (i) This work is licensed under a Creative Commons Attribution 4.0 International License. Read Full License 


\section{Abstract}

\section{Background:}

Subdural empyema is the collection of pus beneath the dura matter, commonly manifests as a complication of oto-rhino-laryngeal infection and rarely locates in the inter-hemispheric subdural space either as an isolated collection or as extension from the convexity of the cerebral hemisphere.

\section{Case presentation:}

A case of right fronto-parietal convexity subdural empyema with inter-hemispheric extension in 19 years old male is presented patient presented with fever, left sided convulsions, hemiparesis and blurring of vision. Despite the use of broad spectrum antibiotics based on cultures, surgical intervention with craniotomy was the definitive intervention needed for good recovery.

\section{Conclusion:}

Subdural empyema located in inter-hemispheric area represents rare form of subdural empyema. The best investigation to choose to diagnose these lesions is definitely magnetic resonance imaging of the brain .craniotomy is the surgical intervention of choice in most of the cases and almost lead to complete evacuation of the collection followed by the use of empirical antibiotic for six weeks duration, both means of treatment are used in order to reach better functional outcome.

\section{Background}

Subdural empyema is a localized collection of purulent material between the dura and arachnoids matter [1], with 95\% of reported cases located within the cranium (mostly involving the frontal lobe).

It causes clinical problems through extrinsic compression and inflammatory reaction involving the brain and meanings, Usually it is unilateral [2] ,with different causes related to different age groups, in infants and young children, subdural empyema most often occurs as a complication of meningitis[3] ,while in adults it is due to Para-nasal sinusitis[4] ,otitis media or mastoiditis, reported cases due to pulmonary tuberculosis [5], falciparum malariae[6, 7] or dental extraction [8]are also present but they are very uncommon .trauma and previous surgeries also considered as a major predisposing factors .

The knowledge of the radiological findings of subdural empyema impacted early diagnosis and lessened dramatically the mortality and morbidity related to this rare condition.

patients usually present with a triad of fever, headache and vomiting and many other symptoms related to the site and size of the collection, most common causative organisms are anaerobic and microaerophilic streptococci [1]. Surgical evacuation through burr-holes or craniotomy is the treatment of choice with empirical antibiotics for 3-6 weeks based on cultures and sensitivity of the isolated purulent material. The pathology, clinical presentation, diagnostic evaluation and methods pre and post operatively, and treatment of subdural empyema were reviewed.

\section{Case Presentation}

A 19 years old male presented to emergency department with headache, high grade fever and vomiting for one week. Two days prior to admission patient developed left side weakness, blurring of vision and generalized tonic-clonic convulsions. Patient was diagnosed as malaria case a week prior to admission, received artemether injection with no improvement; patient had no previous history of head injury or otolaryngeal infection.

On examination patient looks generally ill and confused, with normal vital signs readings apart from raised temperature (38.4 C). GCS 14/15, right pupil was fixed and fully dilated, other cranial nerves were intact, left side hemiplegia, neck stiffness with negative other signs of meningeal irritation. Leukocyte count was 20,100; C- reactive protein was $183.6 \mathrm{mg} / \mathrm{dl}$. CT scan of the brain showed right front parietal subdural empyema with inter-hemispheric extension [Figure 1].

Ophthalmology, dentistry and oto-rhino-laryngologist departments were consulted to exclude any predisposing factors related to his diagnosis that may need further workup and management but no acute or chronic pathology found that can be complicated with such presentation.

Empirical antibiotics (meropenum, vancomycin and metronidazole) with urgent surgical evacuation with burr-holes were performed, evacuated pus sent for culture and sensitivity. Streptococcus viridians isolated which was sensitive to meropenum.

Soon after surgery patient developed another attack of generalized tonic-clonic convulsion began focally at the left side associated with acute confusion state; follow up CT scan showed mainly expansion of the inter-hemispheric part necessitated surgical evacuation through parietal craniotomy [Figure 2]. 


\section{Results}

Patient improved gradually after the second surgery, continued on intravenous antibiotics for 6 weeks after surgery with gradual resolution of symptoms. Patient Discharged fully conscious with complete recovery of his symptoms and signs and his 4 weeks follow up CT brain showed minimal residual of the inter-hemispheric part [Figure 3].

Patient regular visits to the outpatient clinic 1, 3 and 6 months after surgery showed complete neurological recovery.

\section{Discussion And Conclusion}

Authors reported a case of right fronto-parietal subdural empyema with inter-hemispheric extension that responded well with the concomitant use of empirical antibiotics with surgical evacuation based on clinical presentation and radiological diagnostic tools that were crucial for the fast precise diagnosis.

Gender, general characteristics and findings of our case were consistent with the male predominance related to subdural empyema, the common unilateral origin and fronto-parietal convexity site reported [9]. Once infection is established in the subdural space, progression of an empyema tends to be remarkably abrupt. The exudates can spread extensively throughout the subdural space [10]. Patient age and results of isolated cultures (streptococcus viridians) prove the strong relation between odonto-sinosidal infections as the major cause of CNS complications [8] especially in this age group, although no previous history was relevant.

Presented case showed the classical triad of symptoms related to subdural hematoma which is known to be nonspecific and more related to his previous diagnosis of malaria, but his unilateral left side hemiparesis, the right side fixed dilated pupil and focal convulsions are regarded as main symptoms of inter-hemispheric subdural empyema [11].

Subdural empyema cases due to falciparum malaria are rarely present [6, 7] but all have negative bacterial cultures in common, and this fact force us to exclude malaria as the primary cause in our situation.

Once the suspicion of CNS infection is made, patient should be given empirical high dose of antibiotics able to penetrate the cerebrospinal fluids and capable of eradicating the most common causative organisms [1], in our case vancomycin, metronidazole and meropenum were used and proven to be sensitive.

In brain CT ,SDE related to brain convexity appear as Crescent or lentiform extra axial hypo-dense collection with medial border rim enhancement while in inter-hemispheric SDE it tend to be convex on its lateral border and flat on the medial border which is formed by the falx [10].

MRI with gadolinium contrast is the diagnostic tool of choice with hypo-intense area on T1, hyper-intensity on T2 with peripheral contrast enhancement and diffusion restriction on diffusion weighted imaging (DWI) [12] ,making it more informative and sensitive than CT scan especially with DWI sequence.

surgical evacuation with bur-holes as our primary urgent intervention and the need for craniotomy three days after duo to progression of symptoms proves and highly recommend craniotomy as the first definitive intervention, and that was the same suggestion reported by a case series in Australia conducted for 10 years duration [9] and agreed upon by many reported cases especially with inter-hemispheric type as shown in [Table.1] reviewing the latest 10 years .during the operation ,purulent fluid and granulated tissues must be removed except for the thin capsule that can be adherent to vital structures ,moreover we need to make sure that all the pockets are opened and irrigated profoundly with antibiotics [13]. 
Table 1

Reported cases of interhemispheric subdural empyema in the past 10 years

\begin{tabular}{|c|c|c|c|c|c|c|c|c|c|}
\hline Outcome & $\begin{array}{l}\text { duration } \\
\text { of } \\
\text { antibiotic } \\
\text { therapy** }\end{array}$ & Pus C\&S & Surgery & Origin & GCS & $\begin{array}{l}\text { duration } \\
\text { in days }\end{array}$ & Symptoms & Sex,age* & $\begin{array}{l}\text { Reference } \\
\text { number and } \\
\text { year of } \\
\text { publications }\end{array}$ \\
\hline Good & weeks 6 & No growth & $\begin{array}{l}\text { Burhole } \\
\text { then } \\
\text { craniotomy }\end{array}$ & NA & $14 / 15$ & 30 & $\begin{array}{l}\text { vomiting } \\
\text {,Headache, fever }\end{array}$ & $M, 5$ & 2011 [14] \\
\hline Good & NA & No growth & craniotomy & sinusitis & NA & 14 & $\begin{array}{l}\text { Fever, } \\
\text { vomiting,,seizures } \\
\text { „monoparesis }\end{array}$ & $16, \mathrm{M}$ & 2015 [15] \\
\hline Good & NA & $\begin{array}{l}\text { Streptococcus } \\
\text { entermedius }\end{array}$ & $\begin{array}{l}\text { Burhole } \\
\text { then } \\
\text { Endoscopic } \\
\text { aspiration }\end{array}$ & sinusitis & $13 / 15$ & 3 & $\begin{array}{l}\text { Headache, fever } \\
\text {,hemiparesis } \\
\text { „menengism }\end{array}$ & $13, \mathrm{M}$ & 2016 [16] \\
\hline Good & 3 weeks & No growth & craniotomy & Sinusitis & $15 / 15$ & NA & $\begin{array}{l}\text { Fever ,left } \\
\text { hemiparesis }\end{array}$ & $17, \mathrm{~F}$ & 2016 [17] \\
\hline Good & NA & No growth & $\begin{array}{l}2 \text { stages } \\
\text { craniotomy }\end{array}$ & sinusitis & NA & 2 & $\begin{array}{l}\text { fever, aphasia, } \\
\text { convulsions ,right } \\
\text { hemiparesis }\end{array}$ & $14, F$ & 2017 [18] \\
\hline Good & NA & No growth & craniotomy & $\begin{array}{l}\text { Allergic } \\
\text { rhinitis }\end{array}$ & NA & 10 & $\begin{array}{l}\text { hemiparesis } \\
\text {,speech difficulty }\end{array}$ & $17, M$ & 2017 [19] \\
\hline NA & NA & NA & craniotomy & sinusitis & NA & NA & NA & $14, F$ & 2017 [20] \\
\hline \multirow[t]{2}{*}{ Good } & NA & Prevotella oris & craniotomy & Sinonasal & $15 / 15$ & & & $21, F$ & 2019 [21] \\
\hline & & & & & & NA & $\begin{array}{l}\text { convulsions,fever } \\
\text {,hemiparesis }\end{array}$ & & \\
\hline
\end{tabular}

*: Age in Years, GCS: Glasgow coma Scale, C\&S: culture and sensitivity, ** all reported cases were given antibiotics based on culture and sensitivity or even if it came out negative, NA: not available.

In conclusion, sub-dural empyemas with inter-hemispheric extension are very rare, especially in the era of antibiotics, as it's known to be one of the complications of sinusitis being the as the commonest cause, and also it might be as a complication of other pathologies such as meningitis, otitis media, malaria, post-cranial surgery and it might be due to bacterial seeding of sub-dural hematoma. In our case there was no obvious cause. The initial management was a right side double burr-hole craniostomy which was sufficient to evacuate the convexity part of the subdural empyema, but the progression of the patient's condition three days postoperatively necessitate a second surgery in the form of parietal craniotomy and complete evacuation of the empyema including the inter-hemispheric part of the subdural empyema extension. In our opinion the use of craniotomy coupled with culture and sensitivity based intravenous antibiotics appears to grant the best modality in managing such condition.

\section{Abbreviations}

\section{GCS}

Glasgow coma scale, CT:computed tomography, MRI:magnetic resonance Imaging,

SDE

subdural Empyema, DWI:diffusion weighted image, CNS:central nervous system

\section{Declarations}

\section{Ethics approval and consent to participate}

Ethical approval was obtained from the ethical committee of Aliaa specialist hospital. 
Written informed consent was obtained from the patient for publication of this case report and accompanying images and it is available for review by editors of this journal.

\section{Availability of data and material}

All data generated or analyzed during this study are included in this published article and its supplementary information files.

\section{Competing interests}

The authors declare that they have no competing interests.

\section{Funding}

The authors received no financial support for the research, authorship, and/or publication of this article

\section{Authors' contributions}

Moayad Ahmed operated on the patient, collected the data concerning the history, clinical examination and follow ups, adjusted the manuscript to the final form and was a major contributor.

Shahd Altayeb reviewed the literature, wrote the case presentation, discussion and adjusted the manuscript to the final form and was a major contributor.

Zakaria Mohammed operated on the patient, reviewed the data, revised the manuscript, contribute to discussion and conclusion.

Mukashfi Ali revised the manuscript, contribute to conclusion.

All authors read and approved the final manuscript.

\section{Acknowledgements}

none.

\section{References}

1. John E. Greenlee.CNS infections,Subdural Empyema, Current Treatment Options in Neurology 2003, 5:13-2.

2. Segun Toyin Dawodu. Subdural empyema. Emedicine.medscape.com. N.p., 2017. Web. Nov 27, 2017

3. Rahmati MB, Mamishi S, Rezai MS, Houshmandi MM. Subdural Empyema (Case Report). Asian J Med Pharm Res. 2013;3(3):74-7.

4. Martines F, Salvago P, Ferrara S, Mucia M, Gambino A, Sireci F. Parietal subdural empyema as complication of acute odontogenic sinusitis: a case report. Journal of medical case reports. 2014;8:282. https://doi.org/10.1186/1752-1947-8-282.

5. Banerjee AD: Pandey P. : S. Ambekar : B. A. Chandramouli .Pediatric intracranial subdural empyema caused by Mycobacterium tuberculosis-a case report and review of literature Childs Nerv Syst (2010) 26:1117-1120 DOI 10.1007/s00381-010-1157-3.

6. Pallangyo P, Lyimo F, Nicholaus P, Kain U, Janabi M. Spontaneous Subdural Empyema Following a High-Parasitemia Falciparum Infection in a 58-Year-Old Female From a Malaria-Endemic Region: A Case Report. J Investig Med High Impact Case Rep. 2016;4(3):2324709616666567. https://doi.org/10.1177/2324709616666567.

7. DWARAKANATH, et al : EMPYEMA IN FALCIPARUM MALARIA.J Vect Borne Dis 41, September \& December $2004, \mathrm{pp} 80-82$.

8. Moazzam AA, et al. Intracranial bacterial infections of oral origin /. Journal of Clinical Neuroscience. 2015;22:800-6. http://dx.doi.org/10.1016/j.jocn.2014.11.015.

9. French H, Schaefer N, Keijzers G, Barison D, Olson S. Intracranial subdural empyema: a 10-year case series. The Ochsner journal. 2014;14(2):188-94.

10. Zimmerman RD, Leeds. NE,Danziger A. subdural empyema: CT findings ,radiology 1984;417-422.

11. Interhemispheric Subdural Empyema GGRAEF, Frowein RA et al, editors, Head Injuries. Tumors of the Cerebellar Region@ Springer-Verlag Berlin Heidelberg 19. 
12. Shen YY, Cheng ZJ, Chai JY, Dai TM, Luo Y, Guan YQ, Nie HB. Interhemispheric Subdural Empyema Secondary to Sinusitis in an Adolescent Girl. Chin Med J. 2018;131:2989-90.

13. Yende AK, Mohanty S. Massive falx cerebri empyema. Neurol India. 2003;51:65-6.

14. Salunke PS, Malik V, Kovai P, Mukherjee KK. Falcotentorial subdural empyema: Analysis of 10 cases. Acta Neurochir (Wien). 2011;153:164-9.

15. Kazemi KA, Pishjoo M, Safdari Z. Interhemispheric subdural empyema in 16 years old boy, a case report. Int J Med Invest. 2015;4:407-9.

16. Sammartino F, Feletti A, Fiorindi A, Mazzucco GM, Longatti P. Aspiration of parafalcine empyemas with flexible scope. Childs Nerv Syst. 2016;32:1123-9.

17. Yüksel MO, Gürbüz MS, Karaarslan N, Caliskan T. Rapidly progressing interhemispheric subdural empyema showing a three-fold increase in size within 12 hours: Case report. Surg Neurol Int. 2016;7:872-5.

18. Yakar F, Dogan I, Ozgura O, Eroglu U, Beton S, Unlu A. Subdural empyema as a rare complication of sinonasal infection: a case report and review of literature. Int Surg J. 2017;4:3519-22.

19. Arifianto MR, Ma'ruf AZ, Ibrahim A, Bajamal AH. Interhemispheric and infratentorial subdural empyema with preseptal cellulitis as complications of sinusitis: A case report. Pediatr Neurosurg. 2018;53:128-33.

20. Akhaddar A, editor Cranial subdural empyemas. Atlas of Infections in Neurosurgery and Spinal Surgery. 1st ed. Switzerland: Springer International Publishing; 2017. pp. 51-64.

21. Prieto R, Ortega C. Parafalcine subdural empyema: The unresolved controversy over the need for surgical treatment. Surg Neurol Int. 2019;10:203.

\section{Figures}

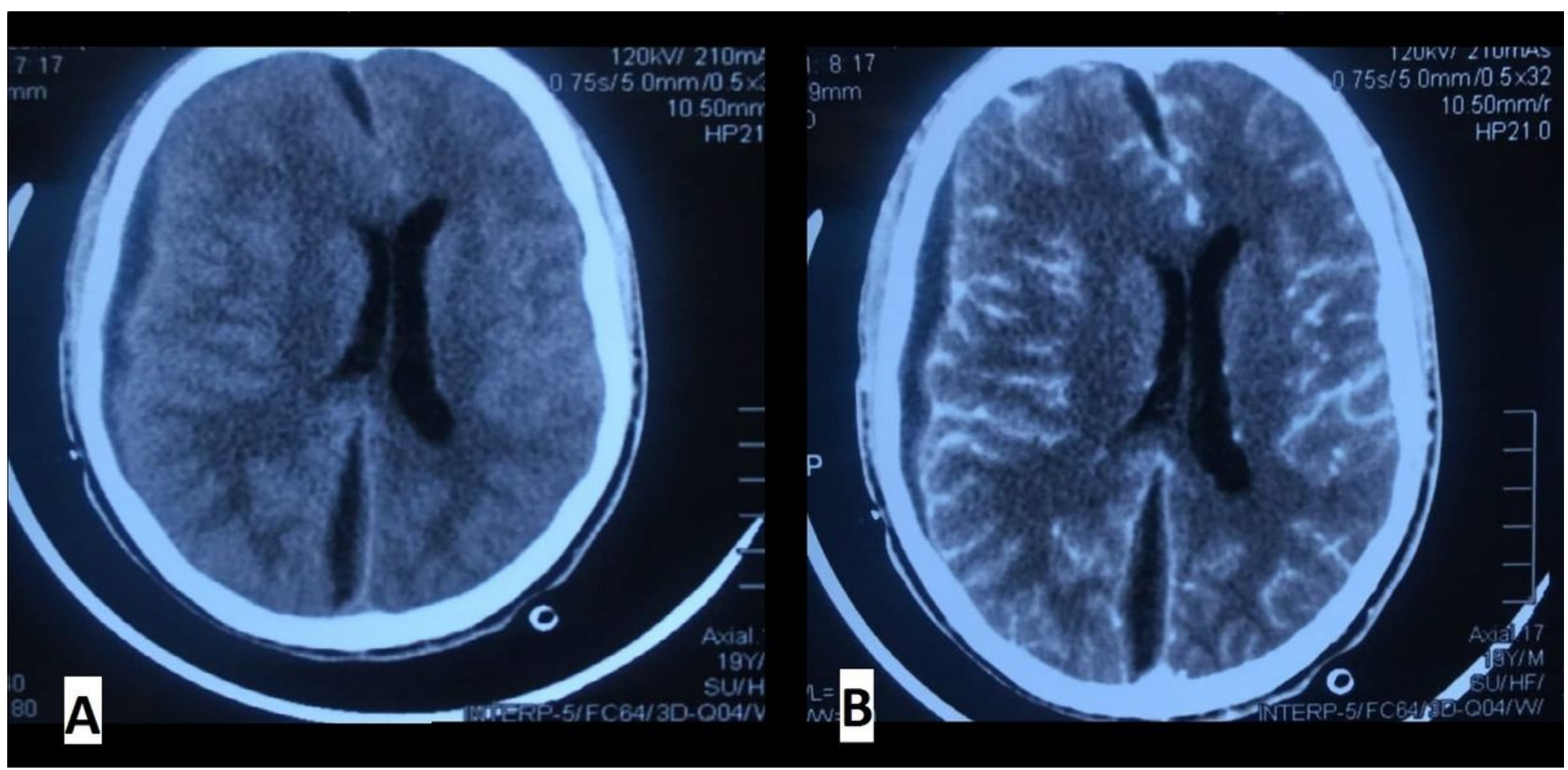

\section{Figure 1}

CT brain. A) non-contrasted axial cut showed right fronto-parieto-occipital convexity subdural collect with inter-hemispheric extension. B) Contrasted axial scan showed enhancing wall around the collection, in both images there is significant mass effect and mid-line shift. 


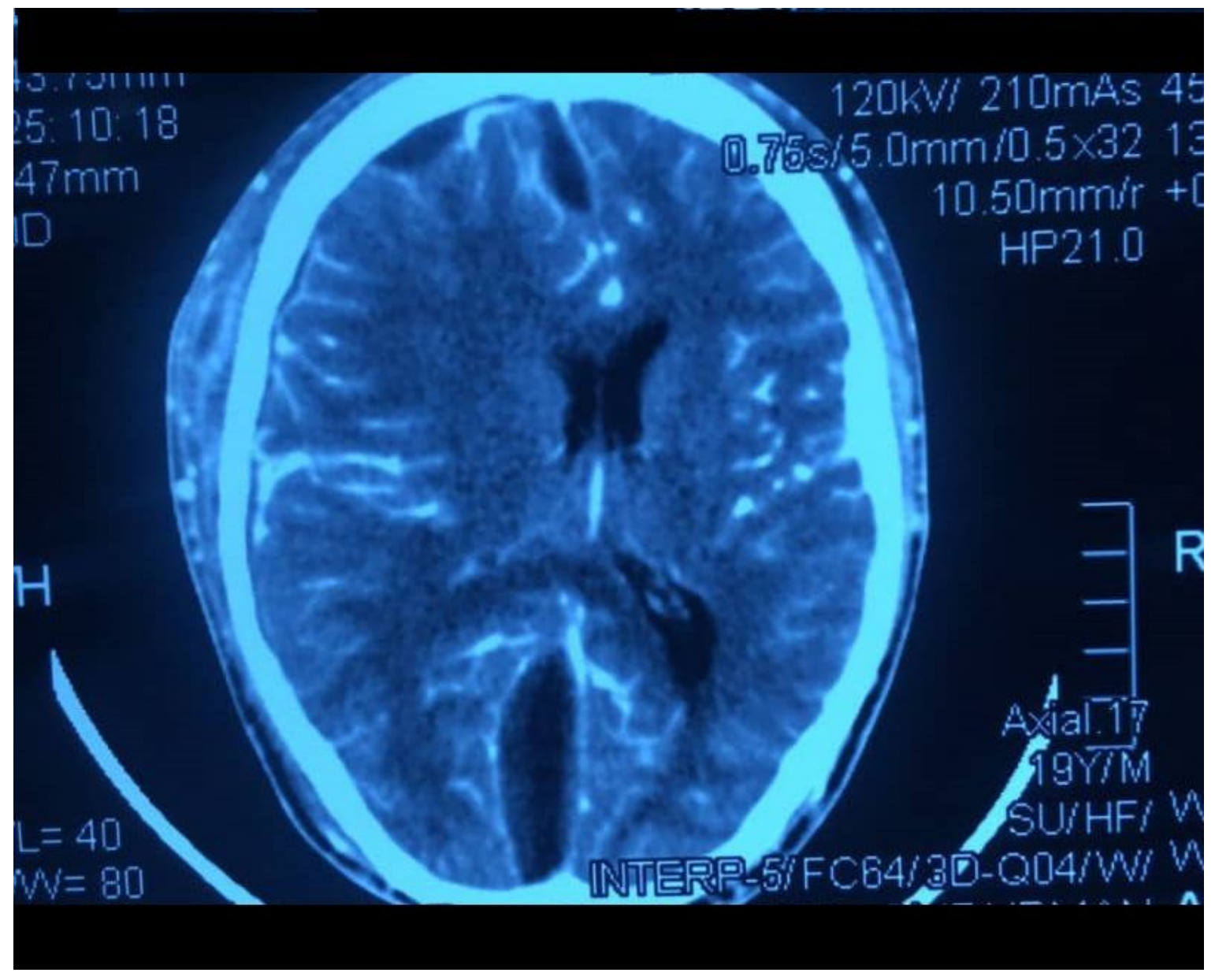

Figure 2

Post 1st surgery CT scan for follow up showed almost resolution of the convexity collection and significant increase in volume of interhemispheric collection.

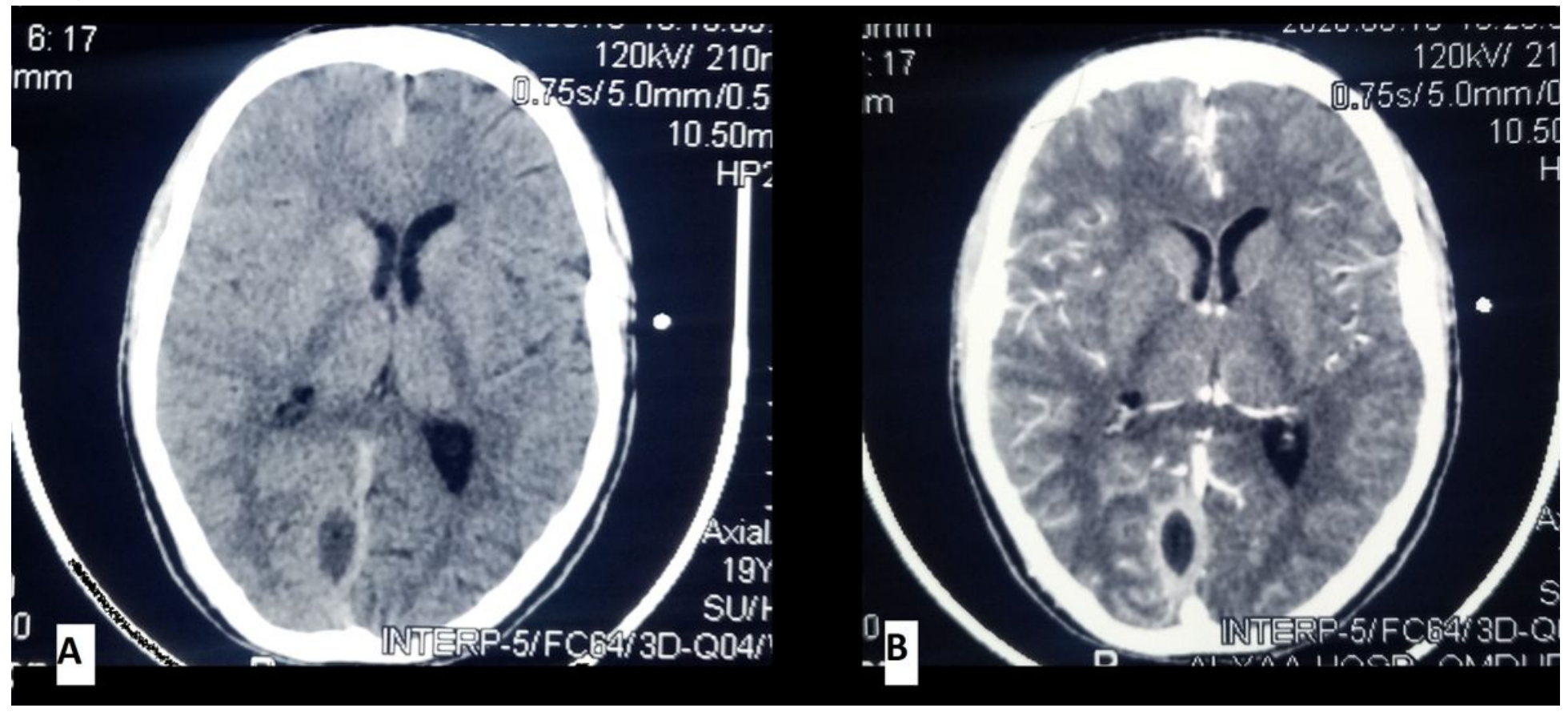

Figure 3

Follow up CT brain taken after the 2nd surgery showed almost total evacuation of the empyema with minimal inter-hemispehric residual 